\title{
Solving the Traveling Salesman's Problem Using the African Buffalo Optimization
}

\author{
Julius Beneoluchi Odili and Mohd Nizam Mohmad Kahar \\ Faculty of Computer Systems \& Software Engineering, Universiti Malaysia Pahang, 26300 Kuantan, Malaysia \\ Correspondence should be addressed to Julius Beneoluchi Odili; odili_julest@yahoo.com
}

Received 16 April 2015; Revised 2 August 2015; Accepted 27 August 2015

Academic Editor: Hong Man

Copyright ( 2016 J. B. Odili and M. N. Mohmad Kahar. This is an open access article distributed under the Creative Commons Attribution License, which permits unrestricted use, distribution, and reproduction in any medium, provided the original work is properly cited.

\begin{abstract}
This paper proposes the African Buffalo Optimization (ABO) which is a new metaheuristic algorithm that is derived from careful observation of the African buffalos, a species of wild cows, in the African forests and savannahs. This animal displays uncommon intelligence, strategic organizational skills, and exceptional navigational ingenuity in its traversal of the African landscape in search for food. The African Buffalo Optimization builds a mathematical model from the behavior of this animal and uses the model to solve 33 benchmark symmetric Traveling Salesman's Problem and six difficult asymmetric instances from the TSPLIB. This study shows that buffalos are able to ensure excellent exploration and exploitation of the search space through regular communication, cooperation, and good memory of its previous personal exploits as well as tapping from the herd's collective exploits. The results obtained by using the ABO to solve these TSP cases were benchmarked against the results obtained by using other popular algorithms. The results obtained using the African Buffalo Optimization algorithm are very competitive.
\end{abstract}

\section{Introduction}

The growing need for profit maximization and cost minimization has never been greater in human history than what we have today. This need has made optimization a very favoured area of scientific investigations. This development has led to the design of a number of optimization algorithms. Some of the most popular algorithms are the Particle Swarm Optimization [1], Ant Colony Optimization [2], Genetic Algorithm [3], Artificial Bee Colony [4], and many others. However, the above algorithms do have some drawbacks ranging from premature convergence [5], delay in obtaining results, easily being stuck in local minima, and complicated fitness function to having many parameters that require setting up [6]. An attempt to proffer solutions to some of the weaknesses of these algorithms is the motivation for the development of the African Buffalo Optimization (ABO).

The $\mathrm{ABO}$ is a population-based stochastic optimization technique that has its inspiration from the behaviour of African buffalos: a species of wild cows, similar to their domestic counterparts that navigate their way through thousands of kilometres in the African rain forests and savannahs by moving together in a large herd of, sometimes, over a thousand buffalos. Their migration is inspired by their search for lush grazing lands. They tend to track the movement of the rainy seasons when they can get lush grazing pastures. As the seasons differ from location to location in the vast African landscape, the buffalos are always mobile in pursuit of their target pastures. In $\mathrm{ABO}$, our interest is in how the buffalos are able to organize themselves in searching the solution space with two basic modes of communications, that is, the alarm "waaa" sounds to indicate the presence of dangers or lack of good grazing fields and, as such, asking the animals to explore other locations that may hold greater promise. On the other hand, the alert "maaa" sound is used to indicate favourable grazing area and is an indication to the animals to stay on to exploit the available resources.

The Traveling Salesman Problem. The Traveling Salesman's Problem (TSP) is the problem faced by a salesman who, starting from a particular town, has the assignment of finding the shortest possible round trip through a given set of customer towns or cities. The salesman has a mandate to visit each city once before finally returning to the starting town/city. The 
Travelling Salesman's Problem (TSP) can be represented by a complete weighted graph $G=(V, E)$ with $V$ being the set of $n$ nodes (cities) and $E$ being the set of edges fully connecting the nodes in the graph $G$. In this graph, each edge $E$ is given a weight $d_{i j}$ which represents the distance between cities $i$ and $j$. It may be important to emphasize that the distance between towns/cities may be symmetric (where the distances between the cities are the same in either going or returning from the towns/cities) or asymmetric (where due to some restrictions, possibly, due to one-way lanes or other reasons, the distances of going from city $A$ to city $B$ may not be the same). The basic minimization equation for TSP is given $n$ towns and their coordinates, find an integer permutation $\pi=$ $C_{1}, C_{2}, C_{3}, \ldots, C_{n}$ with $C_{n}$ being the city " $n$ ", our task is to minimize the sum of the cities $[7,8]$. Consider

$$
f(\pi)=\sum_{i=1}^{n-1} d\left(C_{i}, C_{i+1}\right)+d\left(C_{n}, C_{1}\right) .
$$

Here, $d\left(C_{i}, C_{i+1}\right)$ represents the distance between city " $i$ " and city $i+1$ and $d\left(C_{n}, C_{1}\right)$ is the distance of city " $n$ " and city " 1 ." The aim is to find the shortest path between the adjacent cities in the form of a vector of cities.

This paper is organized in this way: Section 1 introduces the African Buffalo Optimization and the Travelling Salesman's Problem; Section 2 presents a brief review of relevant literature; Section 3 discusses the basic flow of the African Buffalo Optimization (ABO) algorithm; Section 4 is concerned with how the ABO solves the TSP; Section 5 deals with the experiments and discussion of the results of the symmetric TSP instances; Section 6 is concerned with the experiments on the asymmetric TSP instances and the discussions of the results; Section 7 examines the performance of ABO vis-à-vis Neural Networks methods; Section 8 draws conclusions on the study.

\section{Literature Review}

Nature-inspired algorithms (NAs) draw their inspiration from the complex but highly organised attributes of nature. Nature-inspired algorithms which are generally stochastic became the hotbed of several researchers due to the inefficiencies of the exact optimization algorithms (as the problem size enlarges) like the Linear Programming, [9], Dynamic Programming [10], finite elements [11], and finite volume methods. In general NAs simulate the interplay and, sometimes, interdependence of natural elements such as plants, animals, and rivers. The most popular class of algorithms among the NAs are the biology-inspired algorithms. A handful of other NAs, however, are inspired by Chemistry or Physics. Some of those algorithms inspired by Chemistry or Physics include Harmony Search (HS) algorithm, Intelligent Water Drop (IWD), Simulated Annealing (SA), and Black Hole (BH) [12]. In this study, our interest is the biologyinspired optimization algorithms.

Biology-inspired algorithms (BAs) can be categorised into three broad classes, namely, Evolutionary Algorithms (EAs) that are inspired by natural evolutions, the Swarm Intelligence (SI) which simulates the collective behavior in plants and animals, and, thirdly, Ecology algorithms which are concerned with simulating the inter- and intracooperative or competitive interactions within the ecosystem [13].

The Evolutionary Algorithms (EAs), generally, simulate the iterative progress comprising growth, development, reproduction, selection, and survival as seen in a population. EAs are concerned with the feasible solutions that emanate iteratively from generation to generation towards the best solution. EAs employ fitness-based selection within the population of solutions in a way that fitter solutions are selected for survival into the next generation of solutions. In this category of EAs are Genetic Programming (GP), Paddy Field Algorithm (PFA), Evolutionary Strategies (ES), Genetic Algorithm (GA), and the Differential Evolution (DE) [12]. It may be necessary to state that experts have different opinions on the classification of Differential Evolution as an Evolutionary Algorithm. The classification of DE as an Evolutionary Algorithm stems from its use of "Evolution" as one of its parameters (just like other EAs). A critical expert may not class Differential Evolution as being bioinspired, actually [14].

The second category of BAs is the Swarm Intelligence (SI) algorithms which are concerned with the collective social behavior of organisms. The motivation of Swarm Intelligence is the collective intelligence of groups of simple agents such as insects, fishes, birds, bacteria, worms, and other animals based on their behavior in real life. As simple as these animals are, they are able to exhibit exceptional intelligence whenever they work collectively as a group. These algorithms track the collective behavior of animals that exhibit decentralized, selforganized patterns in their foraging duties. Examples of these algorithms are the Bee Colony Optimization (BCO), Firefly Algorithm (FFA), Particle Swarm Optimization (PSO), Ant Colony Optimization (ACO), Artificial Bee Colony (ABC), Bacteria Foraging Algorithm (BFA), and so on [15].

The third category of BAs is the Ecology algorithms which are concerned with the numerous inter- or intraspecies competitive or cooperative interactions within the natural ecosystems. The ecosystem is made up of living organisms along with their abiotic environment with which the organisms interact such as water, air, and soil. Cooperation among organisms includes division of labor and represents the core of their sociality. Some of the interactions are cooperative and others are competitive leading to a complex and harmonious balancing within the ecosystem. Algorithms in this category are the PS2O, Invasive Weed Colony Optimization, and biogeography-based optimization [16].

The development of the African Buffalo Optimization $(\mathrm{ABO})$ is in response to the observed lapses in the existing algorithms. The ABO belongs to the Swarm Intelligence class of algorithms based on the social behavior in animals with the aim of achieving greater exploitation and exploration of the search space, ease of use, and faster speed in achieving the optimal result with its use of relatively fewer parameters in solving combinatorial optimization problems.

2.1. Ant Colony Optimization (ACO). Ant Colony Optimization algorithm is a population-based optimization technique developed by Marco Dorigo and has been successfully applied to solve several NP-hard combinatorial optimization 
problems. This algorithm was inspired by the behavior of ant colonies, especially, by their foraging behavior in real life. Usually ants, when leaving their nests, move randomly around the areas surrounding their nests in search for food. If any of the ants come across food, it first collects some pieces of the food and, on its way back to the nest, deposits a chemical substance called pheromones as a way of communicating to its peers that there has been a breakthrough. Other nearby ants, on perceiving the fragrance of the pheromone, understand and move towards the pheromone path. Once they discover the food source, they, in turn, drop fresh pheromones as a way of alerting other ants. In a matter of time, several ants pick this information and are on the pheromone path.

Another interesting part of the ants' behavior is that as they return to the nest, they optimize their route. In a short while, the ants have created a shorter route to the food source than the previous routes. Moreover, in case an obstruction is put on the shorter route, making movements impossible, the ants are able to find another short route among the available options to evade the obstacle. The highlights of this algorithm include tapping into the indirect communication of a colony of (artificial) ants using pheromone trails as a means of communication, tracking their cooperative ability to solve a complex problem, and harnessing their capacity to optimize their routes from the food source to the nest and vice versa.

There have been several modifications of the ant colony algorithms starting from the initial Ant System (AS), to Ant Colony System (ACS), to Min-Max Ant System (MMAS), and then to the Ant Colony Optimization (ACO) algorithms, and so forth [17]. In ACO, a colony of ants in each iteration constructs a solution probabilistically as ant $k$ at node $i$ selects the next node $j$ to move on to. The choice of node is influenced by the pheromone trail value $\tau_{i j}(t)$ and the available heuristic $\eta_{i j}$. In TSP, $\eta_{i j}=1 / d_{i j}$. So an ant moves from location $i$ to location $j$ with the probability

$$
P_{i j}^{t}(t)= \begin{cases}\frac{\left\lceil\tau_{i j}^{\cdot}(t)\right]^{\alpha} x\left[\eta_{i j}\right]^{\beta}}{\sum_{l \in \mathcal{N}_{i}\left[\tau_{i j}(t)\right]} \alpha x\left[\eta_{i j}\right]^{\beta}} & \text { if } j \in \mathcal{N}_{i^{k}}, \\ 0, & \text { otherwise. }\end{cases}
$$

Here, $\tau_{i j}(t)$ represents the pheromone trail, $\eta_{i j}$ represents the local heuristic information $t$ represents the iteration, $\mathcal{N}_{i^{k}}$ represents the nodes ant $k$ can go to, and $\alpha$ and $\beta$ are parameters that bias the pheromone trails. By the end of an iteration, the pheromone trail on each edge $i j$ is updated using the following equation:

$$
\tau_{i j}^{\cdot}(t+1)=(1-\rho) x \tau_{i j}^{\cdot}(t)+\Delta \tau_{i j}^{\text {best }}(t) .
$$

In (3), $\tau_{i j}^{\cdot}(t+1)$ represents the pheromone trail in iteration $t+1$; $\rho$ takes any values from 0.1 to 0.9 . The amount of pheromone deposited by the best ant is represented by

$$
\begin{aligned}
& \Delta \tau_{i j}^{\text {. best }}(t) \\
& = \begin{cases}\frac{1}{f}\left(s^{\text {best }}(t)\right) & \text { if the best ant used } i j \text { in iteration } t \\
0, & \text { otherwise. }\end{cases}
\end{aligned}
$$

In (4), $f\left(s^{\text {best }}(t)\right)$ represents cost of the best solution $\left(s^{\text {best }}(t)\right)$.
A critical examination of the Ant Colony Optimization technique of solving optimization problems reveals that there is little or no similarity between the ACO's search mechanism and that of the ABO. This could be due to their application of different search techniques in arriving at solutions: while the ACO employs path construction technique, the ABO favours path improvement search mechanism.

2.2. Particle Swarm Optimization. Particle Swarm Optimization which was inspired by the social behavior of birds flocking or fish schooling is one of the biology-inspired computation techniques developed by Eberhart and Kennedy [18]. This algorithm obtains solutions using a swarm of particles, where each particle represents a candidate solution. When compared to evolutionary computation paradigms, a swarm is similar to a population and a particle represents an individual. In searching for a solution, the particles are flown through a multidimensional search space, where the position of each particle is adjusted according to its own experience and that of its neighbors. The velocity vector drives the optimization process as well as highlighting each particle's experience and the information exchange within its neighborhood. Just like the ABO, PSO has two controlling equations in its search for solution and these are (5) and (6). Consider

$$
v_{i j}^{t+1}=x\left(v_{i j}^{t}+\emptyset_{1} U_{1}\left(b_{i j}^{t}-x_{i j}^{t}\right)+\emptyset_{2} U_{2}\left(b_{(n) i j}^{t}-x_{i j}^{t}\right)\right),
$$

where $v_{i j}^{t+1}$ represents the present velocity, $v_{i j}^{t}$ is the previous velocity, $x$ is the constriction factor, $\emptyset_{1}$ and $\emptyset_{2}$ are the acceleration coefficients, $U_{1}$ and $U_{2}$ are random numbers, $b_{i j}^{t}$ is the individual particles' best position, $x_{i j}^{t}$ is the present position, and $b_{(n) i j}^{t}$ is the swarm's best position. The next equation in PSO that calculates the position of the swarm is

$$
x_{i j}^{t+1}=x_{i j}^{t}+v_{i j}^{t+1} .
$$

In PSO algorithm, the particles move in the domain of an objective function $f: \Theta \in R n$, where $n$ represents the variables to be optimized. Each particle, at a particular iteration, is associated with three vectors:

(a) Present position, denoted by $x$ : This vector records the present position of a particular particle.

(b) Present velocity, denoted by $v$ : This vector stores the particle's direction of search.

(c) Individual's best position, denoted by $b$ : This vector records the particular particle's best solution so far since the search began (since the beginning of the algorithm's execution). In addition to these, the individual particles relate with the best particle in the swarm which PSO algorithm tracks, in each iteration, to help direct the search to promising areas [19].

2.3. Artificial Bee Colony. This algorithm, which is inspired by the behavior of natural honey bee swarm, was proposed by Karaboga and Akay in 2009 [20]. It searches for solution through the use of three classes of bees: scout bees, onlooker bees, and employed bees. Scout bees are those that fly over 
the search space in search for solutions (food source). The onlooker bees, on the other hand, are the ones that stay in the nest waiting for the report of the scout bees while the employed bees refer to the class of bees which, after watching the waggle dance of the scout bees, opts to join in harvesting the food source (exploitation). A particular strength of this algorithm lies in its bee transformation capabilities. For instance, a scout bee could transform to an employed bee once it (the same scout bee) is involved in harvesting the food source and vice versa.

Generally, the bees can change their statuses depending on the needs of the algorithm at a particular point in time. In this algorithm, the food source represents a solution to the optimization problem. The volume of nectar in a food source represents the quality (fitness) of the solution. Moreover, each employed bee is supposed to exploit only one food source, meaning that the number of employed bees is the same as the number of food sources. The scout bees are always exploring for new food sources $\vec{v}_{m}$ with higher nectar quantity and/or quality (fitness) $\vec{x}_{m}$ within the neighbourhood. The bees evaluate the nectar fitness using

$$
v_{m i}=x_{m i}+\emptyset_{m i}\left(x_{m i}-x_{k i}\right) \text {, }
$$

where $i$ is a randomly chosen parameter index; $\emptyset_{m i}$ is a random number within a given range; $x_{k i}$ is a food source.

The quality (fitness) of a solution $\mathrm{fit}_{m}\left(\overrightarrow{x_{m}}\right)$ is calculated using the following equation:

$$
\text { fit }_{m}\left(\overrightarrow{x_{m}}\right)= \begin{cases}\frac{1}{1+f_{m}\left(\overrightarrow{x_{m}}\right)} x, & \text { if } f_{m}\left(\overrightarrow{x_{m}}\right)>0 \\ 1+f_{m}\left(\overrightarrow{x_{m}}\right), & \text { if } f_{m}\left(\overrightarrow{x_{m}}\right)<0 .\end{cases}
$$

From the foregoing discussion, it is clear that there is slight similarity between (5) in PSO and (7) in ABO since each algorithm subtracts a variable from the personal and individual bests of the particles/buffalos. For PSO, the subtracted variable is the present position and for $\mathrm{ABO}$, it is the immediate-past explored location (the waaa values, $w . k$ ). However, the two equations are different in several respects: while the PSO uses $x$ (being the constriction factor) or $\omega$ (as inertia factor, in some versions of the PSO), there are no such equivalents in ABO. Moreover, while the PSO employs random numbers $\left(U_{1}\right.$ and $\left.U_{2}\right), \mathrm{ABO}$ does not use random numbers, only learning parameters. Also, PSO uses acceleration coefficients $\left(\emptyset_{1}\right.$ and $\left.\emptyset_{2}\right)$; ABO does not. In the case of the $\mathrm{ABC}$, even though it employs the same search technique in arriving at solutions, the algorithm procedures are quite different.

2.4. Information Propagation. In searching for solutions to an optimization problem, the ACO employs the path construction technique while the $\mathrm{PSO}, \mathrm{ABC}$, and the $\mathrm{ABO}$ use the path improvement technique. However, while the PSO uses the Von Neumann (see Figure 1) as its best technique for information propagation [21], the ACO obtains good results using the ring topology [22] and the ABO uses the star topology which connects all the buffalos together. The Von Neumann topology enables the particles to connect to neighboring particles on the east, west, north, and south. Effectively, a particular particle relates with the other four particles surrounding it. The ABO employs the star topology such that a particular buffalo is connected to every other buffalo in the herd. This enhances ABO's information dissemination at any particular point in time.

\section{African Buffalo Optimization Algorithm}

In using the $\mathrm{ABO}$ to proffer solutions in the search space, the buffalos are first initialized within the herd population and are made to search for the global optima by updating their locations as they follow the current best buffalo bgmax in the herd. Each buffalo keeps track of its coordinates in the problem space which are associated with the best solution (fitness) it has achieved so far. This value is called $b p$ max. $k$ representing the best location of the particular buffalo in relation to the optimal solution. The $\mathrm{ABO}$ algorithm follows this pattern: at each step, it tracks the dynamic location of each buffalo towards the bpmax. $k$ and $b g$ max depending on where the emphasis is placed at a particular iteration. The speed of each animal is influenced by the learning parameters.

3.1. ABO Algorithm. The $\mathrm{ABO}$ algorithm is presented below:

(1) Initialization: randomly place buffalos to nodes at the solution space.

(2) Update the buffalos fitness values using

$$
\begin{aligned}
m \cdot k+1= & m \cdot k+l p 1(\text { bgmax }-w \cdot k) \\
& +l p 2(b p \max . k-w \cdot k)
\end{aligned}
$$

where $w . k$ and $m . k$ represent the exploration and exploitation moves, respectively, of the $k$ th buffalo $(k=1,2, \ldots, N) ; l p 1$ and $l p 2$ are learning factors; bgmax is the herd's best fitness and bpmax.k the individual buffalo $k$ 's best found location.

(3) Update the location of buffalo $k$ (bpmax.k and $b g$ max) using

$$
w \cdot k+1=\frac{(w \cdot k+m \cdot k)}{ \pm 0.5}
$$

(4) Is bgmax updating? Yes, go to (5). No, go to (2).

(5) If the stopping criteria are not met, go back to algorithm step (3); else go to (6).

(6) Output best solution.

A closer look at the algorithm (the ABO algorithm) reveals that (9) which shows the democratic attitude of the buffalos has three parts: the first $m . k$ represents the memory of the buffalos past location. The buffalo has innate memory ability that enables it to tell where it has been before. This is crucial in its search for solutions as it helps it to avoid areas that produced bad results. The memory of each buffalo is a list of solutions that can be used as an alternative for the current local maximum location. The second $l p 1(b g \max -w \cdot k)$ is 


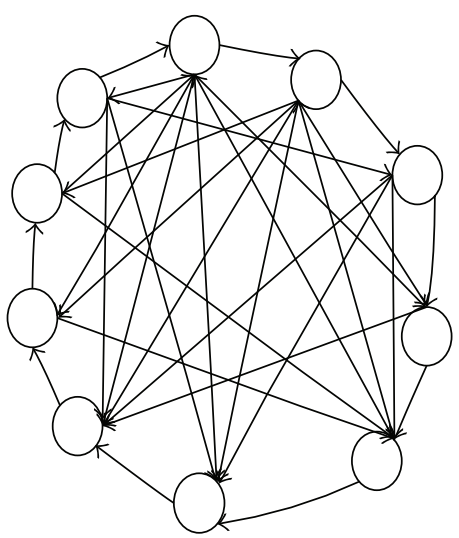

Star topology

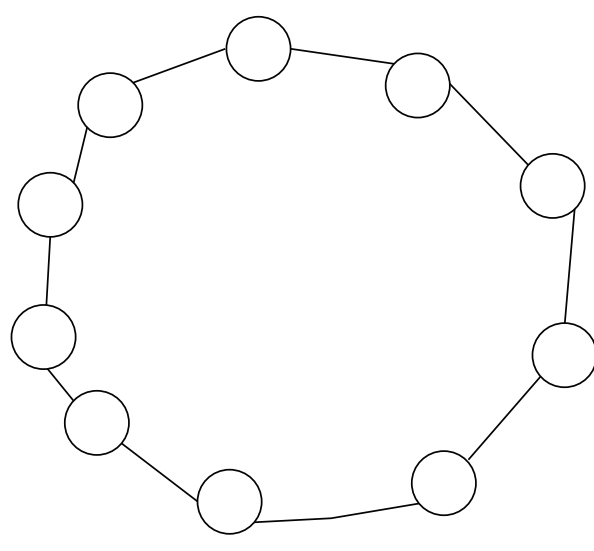

Ring topology

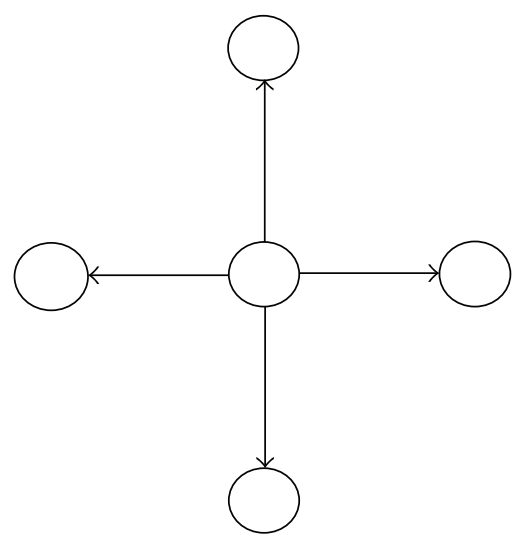

Von Neumann topology

FIGURE 1: Information propagation topologies.

concerned with the caring or cooperative part of the buffalos and is a pointer to the buffalo's social and informationsharing experience and then the third part $l p 2(b p \max . k-w . k)$ indicates the intelligence part of the buffalos. So the $\mathrm{ABO}$ exploits the memory, caring intelligent capabilities of the buffalos in the democratic equation (9). Similarly, (10) is the waaa vocalization equation that propels the animals to move on to explore other environments as the present area has been fully exploited or is unfavourable for further exploration and exploitation.

\subsection{Highlights of the ABO Algorithm. They are as follows:}

(1) Stagnation handling through regular update of the best buffalo bgmax in each iteration.

(2) Use of relatively few parameters to ensure speed fast convergence.

(3) A very simple algorithm that require less than 100 lines of coding effort in any programming language.

(4) Ensuring adequate exploration by tracking the location of best buffalo (bgmax) and each buffalo's personal best location (bpmax.k).

(5) Ensuring adequate exploitation through tapping into the exploits of other buffalos lp $1(b g \max -w \cdot k)$.

3.3. Initialization. The initialization phase is done by randomly placing the $k$ th buffalo in the solution space. For initialization, some known previous knowledge of the problem can help the algorithm to converge in less iterations.

3.4. Update Speed and Location. In each iteration, each buffalo updates its location according to its former maximum location (bpmax) and some information gathered from the exploits of the neighboring buffalos. This is done using (9) and (10) (refer to the ABO algorithm steps (2) and (3) above). This enables the algorithm to track the movement of the buffalos in relation to the optimal solution.

\section{Using ABO to Solve the Travelling Salesman's Problem}

The ABO has the advantage of using very simple steps to solve complex optimization problems such as the TSP. The basic solution steps are as follows:

(a) Choose, according to some criterion, a start city for each of the buffalos and randomly locate them in those cities. Consider

$$
\begin{aligned}
P a b & =\frac{w^{l p 1} a b m^{l p 2} a b}{\sum_{i=1}^{n} w^{l p 1} a b m^{l p 2} a b} \\
a b & = \pm 0.5 .
\end{aligned}
$$

(b) Update buffalo fitness using (9) and (10), respectively.

(c) Determine the bpmax. $k$ and max.

(d) Using (11) and heuristic values, probabilistically construct a buffalo tour by adding cities that the buffalos have not visited.

(e) Is the bgmax updating? Go to (f). No, go to (a).

(f) Is the exit criteria reached? Yes, go to (g). No, return to (b).

(g) Output the best result.

Here, $l p 1$ and $l p 2$ are learning parameters and are 0.6 and 0.4 , respectively, $a b$ takes the values of 0.5 and -0.5 in alternate iterations, $m$ is the positive reinforcement alert invitation which tells the animals to stay to exploit the environment since there are enough pastures, and $w$ is the negative reinforcement alarm which tells the animals to keep on exploring the environment since the present location is not productive. For buffalo $k$, the probability $p^{k}$ of moving from city $j$ to city $k$ depends on the combination of two values, namely, the desirability of the move, as computed by some heuristic indicating the previous attractiveness of that move and the summative benefit of the move to the herd, indicating how productive it has been in the past to make that particular move. The denominator values represent an indication of the desirability of that move. 
TABLE 1: TSPLIB datasets.

\begin{tabular}{lcccc}
\hline 1st experimental datasets & 2nd experimental datasets & ATSP dataset & \multicolumn{2}{c}{ NN comparative datasets } \\
\hline Berlin52 & Att48 & R48p & Eil51 & KroA100 B \\
St70 & St70 & Ft70 & Eil76 & KroA200 \\
Eil76 & Eil76 & Kro124p & Eil101 & KroB100 \\
Pr76 & Pr152 & Ftv70 & Berlin52 & KroB150 \\
Kroa100 & Gil262 & P43 & Bier127 & KroB200 \\
Eil101 & Rd400 & Ftv170 & Ch130 & KroC100 \\
Ch150 & Pr1002 & & Ch150 & KroD100 \\
Tsp225 & D1291 & Rd100 & KroE100 \\
& Fnl4461 & Lin105 & Rat575 \\
& Brd14051 & Lin318 & RL1323 \\
\hline
\end{tabular}

4.1. ABO Solution Mechanism for the TSP. The ABO applies the Modified Karp Steele algorithm in its solution of the Travelling Salesman's Problem [23]. It follows a simple solution step of first constructing a cycle factor $F$ of the cheapest weight in the $K$ graph. Next, it selects a pair of edges taken from different cycles of the $K$ graph and patch in a way that will result in a minimum weight. Patching is simply removing the selected edges in the two cycle factors and then replacing them with cheaper edges and in this way forming a larger cycle factor, thus, reducing the number of cycle factors in graph $K$ by one. Thirdly, the second step is repeated until we arrive at a single cycle factor in the entire graph $K$. This technique fits into the path improvement technique description [24]. ABO overcomes the problem of delay in this process through the use of two primary parameters to ensure speed, namely, $l p 1$ and $l p 2$, coupled with the algorithm keeping a track of the route of the $b g$ max as well as $b p$ max. $k$ in each construction step.

\section{Experiments and Discussion of Results}

In this study, the $\mathrm{ABO}$ was implemented on three sets of symmetric TSP datasets and a set of asymmetric TSP (ATSP) ranging from 48 to 14461 cities from TSPLIB95 [25]. The first experiment was concerned with the comparison of the performance of $\mathrm{ABO}$ in TSP instances with the results obtained from a recent study [26] involving Berlin52, St70, Eil76, Pr76, KroA100, Eil101, Ch150, and Tsp225. The second set of experiments was concerned with testing ABO's performance with another recently published study [27] on Att48, St70, Eil76, Pr152, Gil262, Rd400, Pr1002, D1291, Fn14461, and Brd14051. The third experiment examined ABO's performance in asymmetric TSP instances. The fourth set of experiments involved comparing $\mathrm{ABO}$ results with those obtained using some popular Artificial Neural Networks methods [28]. The TSP benchmark datasets are presented in Table 1.

5.1. Experimental Parameters Setting. For the sets of experiments involving PSO, the parameters are as follows: population size: 200; iteration $\left(T_{\max }\right): 1000$; inertia weight: 0.85 ; $C_{1}: 2 ; C_{2}: 2 \operatorname{rand} 1(0,1) \operatorname{rand} 2(0,1)$. For the HPSACO, the experimental parameters are as follows: population: 200; iteration $\left(T_{\max }\right): 1000$; inertia weight: $0.85 ; C_{1}: 2 ; C_{2}: 2$; ants
$(N)$ : 100; pheromone factor $(\alpha)$ : 1.0 ; heuristic factor $(\beta): 2.0$; evaporation coefficient $(\rho)$ : 0.05 ; pheromone amount: 100 . For the experiments involving other algorithms, in this study, Table 2 contains the details of the parameters used.

5.2. Experiment Analysis and Discussion of Results. The experiments were performed using MATLAB on Intel Duo Core i7-3770 CPU, $3.40 \mathrm{ghz}$ with 4 GB RAM. The experiments on the asymmetric Travelling Salesman's Problems were executed on a Pentium, Duo Core, $1.80 \mathrm{Ghz}$ processor and 2 GB RAM desktop computer. Similarly, the experiments on the Artificial Neural Networks were executed using Microsoft Virtual C++, 2008, on an Intel Duo Core, i7, CPU. To authenticate the veracity of the $\mathrm{ABO}$ algorithm in solving the TSP, we initially carried out experiments on eight TSP cases. The city-coordinates data are available in [29]. The results are presented in Table 3.

In Table 3, the "Average Value" refers to the average fitness of each algorithm, and the "relative error" values are obtained by calculating

$$
\left(\frac{\text { Average value }- \text { Best value }}{\text { Best value }}\right) \times 100 \text {. }
$$

As can be seen in Table 3, the ABO outperformed the other algorithms in all test instances under investigation. The ABO, for instance, obtained the optimal solution to Berlin52 and Eil76. No other algorithm did. Besides this, the ABO obtained the nearest-optimal solution to the remaining TSP instances compared to any other algorithm. In terms of the average results obtained by each algorithm, the $\mathrm{ABO}$ still has the best performance. It is rather surprising that the Hybrid Algorithm (HA) which uses a similar memory matrix like the $\mathrm{ABO}$ could not pose a better result. This is traceable to the use of several parameters since the HA is a combination of the $\mathrm{ACO}$ and the ABC: the two algorithms that have some of the largest numbers of parameters to tune in order to obtain good solutions.

The dominance of the $\mathrm{ABO}$ can also be seen in the use of computer resources (CPU time) where it is clearly seen that the $\mathrm{ABO}$ is the fastest of all four algorithms. In Berlin52, for instance, the $\mathrm{ABO}$ is 58,335 times faster than the ACO; 1,085 times faster than the $\mathrm{ABC} ; 30,320$ times faster than the Hybrid Algorithm (HA). This trend continues in all the 
TABLE 2: Experimental parameters.

\begin{tabular}{|c|c|c|c|c|c|c|c|c|c|}
\hline \multirow{2}{*}{ Parameter } & \multirow[b]{2}{*}{ Values } & \multicolumn{2}{|c|}{$\mathrm{ACO}$} & \multicolumn{2}{|l|}{$\mathrm{ABC}$} & \multicolumn{2}{|l|}{$\mathrm{HA}$} & \multicolumn{2}{|c|}{ CGAS } \\
\hline & & Parameters & Values & Parameters & Values & Parameters & Values & Parameter & Values \\
\hline Population & 40 & Ants & $D^{*}$ & Population & $D^{*}$ & Population & $D^{*}$ & Generation & 100 \\
\hline$m \cdot k$ & 1.0 & $\beta$ & 5.0 & $\phi i j$ & $\operatorname{rand}(-1,1)$ & $\beta$ & 5.0 & $\beta$ & 2.0 \\
\hline$b g \max / b p \max$ & 0.6 & $\rho$ & 0.65 & $\omega i j$ & $\operatorname{rand}(0,1.5)$ & $\rho$ & 0.65 & $\rho$ & 0.1 \\
\hline$l p 1 / l p 2$ & 0.5 & $\alpha$ & 1.0 & SN & $\mathrm{NP} / 2$ & $\alpha$ & 1.0 & Ro & 0.33 \\
\hline$w . k$ & 1.0 & O & 200 & Limit & $D^{*} \mathrm{SN}$ & $\phi i j$ & $\operatorname{rand}(-1,1)$ & Crossover rate & 1.0 \\
\hline N/A & - & qo & 0.9 & Max cycle number & 500 & $\omega i j$ & $\operatorname{rand}(0,1.5)$ & qo & 0.9 \\
\hline N/A & - & $\mathrm{N} / \mathrm{A}$ & - & Colony & 50 & SN & $\mathrm{NP} / 2$ & $\phi r$ & 0.3 \\
\hline N/A & - & $\mathrm{N} / \mathrm{A}$ & - & N/A & - & Limit & $D^{*} \mathrm{SN}$ & $\phi \rho$ & 0.2 \\
\hline N/A & - & N/A & - & N/A & - & Max cycle number & 500 & $\tau_{\min }$ & $\tau_{\max } / 20$ \\
\hline N/A & - & N/A & - & N/A & - & Colony & 50 & $\tau_{\max }$ & $1-(1-\rho)$ \\
\hline N/A & - & $\mathrm{N} / \mathrm{A}$ & - & N/A & - & Q & 200 & N/A & - \\
\hline N/A & - & N/A & - & N/A & - & qo & 0.9 & N/A & - \\
\hline Total number of runs & s 50 & & 50 & & 50 & & 50 & & 50 \\
\hline
\end{tabular}

TABLE 3: Comparative experimental result.

\begin{tabular}{|c|c|c|c|c|c|c|c|}
\hline Problem & Number of cities & Optima & Method & Best & Mean & Rel. err. (\%) & Time (s) \\
\hline \multirow{4}{*}{ Berlin52 } & \multirow{4}{*}{52} & \multirow{4}{*}{7542} & $\mathrm{ABO}$ & 7542 & 7616 & $0 \%$ & 0.002 \\
\hline & & & $\mathrm{ACO}$ & 7548.99 & 7659.31 & 1.52 & 116.67 \\
\hline & & & $\mathrm{ABC}$ & 9479.11 & $10,390.26$ & 37.72 & 2.17 \\
\hline & & & HA & 7544.37 & 7544.37 & 0.03 & 60.64 \\
\hline \multirow{4}{*}{ St70 } & \multirow{4}{*}{70} & \multirow{4}{*}{675} & $\mathrm{ABO}$ & 676 & 678.33 & 0.15 & 0.08 \\
\hline & & & $\mathrm{ACO}$ & 696.05 & 709.16 & 4.73 & 226.06 \\
\hline & & & $\mathrm{ABC}$ & 1162.12 & 1230.49 & 81.73 & 3.15 \\
\hline & & & $\mathrm{HA}$ & 687.24 & 700.58 & 3.47 & 115.65 \\
\hline \multirow{4}{*}{ Eil76 } & \multirow{4}{*}{76} & \multirow{4}{*}{538} & $\mathrm{ABO}$ & 538 & 563.04 & $0 \%$ & 0.03 \\
\hline & & & ACO & 554.46 & 561.98 & 3.04 & 271.98 \\
\hline & & & $\mathrm{ABC}$ & 877.28 & 931.44 & 70.78 & 3.49 \\
\hline & & & HA & 551.07 & 557.98 & 2.31 & 138.82 \\
\hline \multirow{4}{*}{ Pr76 } & \multirow{4}{*}{76} & \multirow[t]{4}{*}{108159} & $\mathrm{ABO}$ & 108167 & 108,396 & $0.007 \%$ & 0.08 \\
\hline & & & $\mathrm{ACO}$ & $115,166.66$ & $116,321.22$ & 7.55 & 272.41 \\
\hline & & & $\mathrm{ABC}$ & $195,198.9$ & $205,119.61$ & 89.65 & 3.50 \\
\hline & & & HA & $113,798.56$ & $115,072.29$ & 6.39 & 138.92 \\
\hline \multirow{4}{*}{ Kroa100 } & \multirow{4}{*}{100} & \multirow[t]{4}{*}{21282} & $\mathrm{ABO}$ & 21311 & 22163.8 & $0.4 \%$ & 0.00 \\
\hline & & & $\mathrm{ACO}$ & $22,455.89$ & $22,880.12$ & 7.49 & 615.06 \\
\hline & & & $\mathrm{ABC}$ & $49,519.51$ & $53,840.03$ & 152.94 & 5.17 \\
\hline & & & $\mathrm{HA}$ & $22,122.75$ & $22,435.31$ & 5.40 & 311.12 \\
\hline \multirow{4}{*}{ Eil101 } & \multirow{4}{*}{101} & \multirow[t]{4}{*}{629} & $\mathrm{ABO}$ & 640 & 640 & $1.7 \%$ & 0.027 \\
\hline & & & ACO & 678.04 & 693.42 & 7.96 & 527.42 \\
\hline & & & $\mathrm{ABC}$ & 1237.31 & 1315.95 & 104.88 & 5.17 \\
\hline & & & $\mathrm{HA}$ & 672.71 & 683.39 & 6.39 & 267.08 \\
\hline \multirow{4}{*}{ Ch150 } & \multirow{4}{*}{150} & \multirow[t]{4}{*}{6528} & $\mathrm{ABO}$ & 6532 & 6601 & $0.06 \%$ & 0.032 \\
\hline & & & $\mathrm{ACO}$ & 6648.51 & 6702.87 & 2.61 & 1387.65 \\
\hline & & & $\mathrm{ABC}$ & $20,908.89$ & $21,617.48$ & 230.93 & 8.95 \\
\hline & & & $\mathrm{HA}$ & 6641.69 & 6677.12 & 2.21 & 698.61 \\
\hline \multirow{4}{*}{ Tsp225 } & \multirow{4}{*}{225} & \multirow[t]{4}{*}{3916} & $\mathrm{ABO}$ & 3917 & 3982 & 0.03 & 0.09 \\
\hline & & & ACO & 4112.35 & 4176.08 & 8.22 & 4038.75 \\
\hline & & & $\mathrm{ABC}$ & $16,998.41$ & $17,955.12$ & 365.2792 & 16.68 \\
\hline & & & $\mathrm{HA}$ & 4090.54 & 4157.85 & 7.74 & 2037.33 \\
\hline
\end{tabular}


TABLE 4: Comparative optimal results.

\begin{tabular}{lccccccccccccc}
\hline \multirow{2}{*}{ TSP instance } & \multirow{2}{*}{ Optima } & \multicolumn{3}{c}{ ABO } & \multicolumn{3}{c}{ PSO } & \multicolumn{3}{c}{ ACO } & \multicolumn{3}{c}{ HPSACO } \\
& & Best & Avg & Err. \% & Best & Avg & Err. \% & Best & Avg & Err. \% & Best & Avg & Err. \% \\
\hline att48 & 33522 & 33524 & 33579 & 0.16 & 33734 & 33982 & 0.63 & 33649 & 33731 & 0.62 & 33524 & 33667 & 0.16 \\
st70 & 675 & 676 & 678.33 & 0.15 & 691.2 & 702.6 & 2.40 & 685.7 & 694.7 & 1.59 & 680.3 & 698.6 & 0.79 \\
eil76 & 538 & 538 & 563.04 & 0.00 & 572.3 & 589.1 & 6.38 & 550.7 & 560.4 & 2.36 & 546.2 & 558.1 & 1.52 \\
pr152 & 73682 & 73730 & 73990 & 0.07 & 75361 & 75405 & 2.28 & 74689 & 74936 & 1.37 & 74165 & 74654 & 0.66 \\
gil262 & 2378 & 2378 & 2386 & 0.00 & 2513 & 2486 & 5.68 & 2463 & 2495 & 3.57 & 2413 & 2468 & 1.47 \\
rd400 & 15281 & 15301 & 15304 & 5.00 & 16964 & 17024 & 11.01 & 16581 & 16834 & 8.51 & 16067 & 16513 & 5.14 \\
pr1002 & 259045 & 259132 & 261608 & 0.03 & 278923 & 279755 & 7.67 & 269758 & 271043 & 4.14 & 267998 & 269789 & 3.46 \\
d1291 & 50801 & 50839 & 50839 & 0.07 & 53912 & 54104 & 6.12 & 52942 & 53249 & 4.21 & 52868 & 52951 & 4.07 \\
fnl4461 & 182566 & 182745 & 183174 & 0.10 & 199314 & 199492 & 9.17 & 192964 & 194015 & 5.70 & 191352 & 192585 & 4.81 \\
brd14051 & 469385 & 469835 & 479085 & 0.10 & 518631 & 519305 & 10.49 & 505734 & 511638 & 7.74 & 498471 & 503594 & 6.20 \\
\hline
\end{tabular}

test cases under investigation. To solve all the TSP problems here, it took $\mathrm{ABO}$ a cumulative time of 0.279 seconds to ACO's 7456 seconds; ABC's 43.11 seconds; and HA's 3362.27 seconds. The speed of the $\mathrm{ABO}$ is traceable to effective memory management technique since the $\mathrm{ABO}$ uses the path improvement technique as against the ACO that uses the slower path construction method. The difference in speed with the $\mathrm{ABC}$ that uses similar technique with $\mathrm{ABO}$ is due to the ABC's use of several parameters. The speed of the HA could have been affected by the combination of the path construction and path improvement techniques coupled with the use of several parameters.

5.3. $A B O$ and Other Algorithms. Encouraged by the extremely good performance of the $\mathrm{ABO}$ in the first experiments, more experiments were performed and the results obtained compared with those from PSO, ACO, and HPSACO from another recently published study [27]. The HPSACO is a combination of three techniques, namely, the Collaborative Strategy, Particle Swarm Optimization, and the Ant Colony Optimization algorithms. The datasets used in this experiment are from the TSPLIB95 and they are Att48, St70, Eil76, Pr152, Gil262, Rd400, Pr1002, D1291, Fn14461, and Brd14051. The results are presented in Table 4.

Table 4 further underscores the excellent performance of ABO. The ABO obtained the optimal solution to Eil76 and Gil262 and very near-optimal solution in the rest of test cases. It is obvious from the relative error calculations that the $\mathrm{ABO}$ obtained over 99\% accuracy in all the TSP instances here except the difficult rd100 where it obtained 95\%. It is worthy of note that the $95 \%$ accuracy of the $\mathrm{ABO}$ is still superior to the performance of the other comparative algorithms in this TSP instance. The cumulative relative error of the ABO is 5.68\% to the PSO's $61.83 \%$, the ACO's $39.81 \%$, and the HPSACO's $28.28 \%$. Clearly, from this analysis, the ABO has a superior search capability. This is traceable to its use of relatively fewer parameters than most other Swarm Intelligence techniques. The controlling parameters of the $\mathrm{ABO}$ are just the learning parameters ( $l p 1$ and $l p 2)$.

In designing the $\mathrm{ABO}$, the authors deliberately tried to avoid the "Frankenstein phenomena" [30], that is, a case of developing intricate techniques with several parameters/operators that, most times, the individual contributions of some of such parameters/operators to the workings of the algorithm are difficult to pinpoint. The ability to achieve this "lean metaheuristic" design (which is what we tried to do in designing the $\mathrm{ABO}$ ) is a mark of good algorithm design [30].

\section{ABO on Asymmetric TSP Test Cases}

Moreover, we had to experiment on some ATSP test cases to verify the performance of the novel algorithm on such cases. The results obtained from this experiment using ABO are compared with the results obtained from solving some benchmark asymmetric TSP instances available in TSPLIB95 using the Randomized Arbitrary Insertion algorithm (RAI) [31], Ant Colony System 3-opt (ACS), Min-Max Ant System (MMAS), and Iterated Local Search (ILS) which is reported to be one of the best methods for solving TSP problems. These ATSP instances are ry48p, ft70, Kro124p, ftv70, p43, and $\mathrm{ftv} 170$ [32].

Table 5 shows the comparative results obtained by applying five different algorithms to solving a number of benchmark asymmetric TSP instances with the aim of evaluating the performance of the African Buffalo Optimization (ABO) algorithm. The first column lists the ATSP instances as recorded in the TSPLIB; the second column indicates the number of cities/locations/nodes involved in each instance; the third indicates the optimal solutions, then followed by the performances of the different algorithms in at most 50 test runs.

A quick look at the table shows the excellent performance of the ILS and the MMAS with both obtaining $100 \%$ results in the only four cases available in literature that they solved. The ABO performed very well achieving over $99 \%$ in all test cases but one. The ABO has approximately the same performance with RAI and the ACS obtaining about 99\% optimal results in virtually all cases. It is significant to note, however, that the ABO performed better than ACS and RAI in $\mathrm{ft70}$ which was said to be a difficult ATSP instance to solve in spite of its relatively small size [33].

Next, the authors examined the speed of each algorithm to achieve result since one of the aims of the $\mathrm{ABO}$ is to 


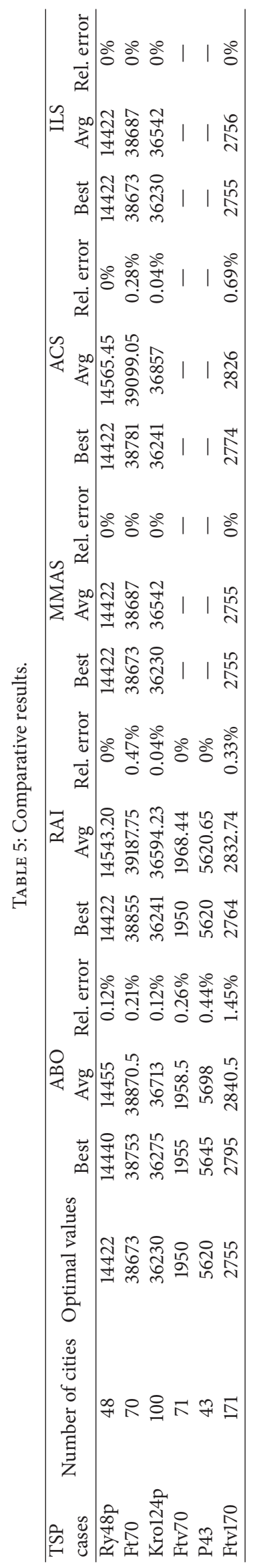


TABLE 6: Comparative speed of algorithms.

\begin{tabular}{|c|c|c|c|c|c|c|}
\hline TSP cases & Number of cities & $\begin{array}{c}\text { ABO } \\
\text { Avg time } \\
(\text { secs })\end{array}$ & $\begin{array}{l}\text { MIMM-ACO } \\
\text { Avg time } \\
\text { (secs) }\end{array}$ & $\begin{array}{l}\text { MMAS } \\
\text { Avg time } \\
\text { (secs) }\end{array}$ & $\begin{array}{c}\text { CGAS } \\
\text { Avg time } \\
(\text { secs })\end{array}$ & $\begin{array}{c}\text { RAI } \\
\text { Avg time } \\
\text { (secs) }\end{array}$ \\
\hline Ry48p & 48 & 0.07 & 7.83 & 7.97 & 12.35 & 1.598 \\
\hline Ft70 & 70 & 0.05 & 9.85 & 10.15 & 15.32 & 7.068 \\
\hline Kro124p & 100 & 0.08 & 33.25 & 23.4 & 78.52 & 30.34 \\
\hline Ftv70 & 71 & 0.09 & 64.53 & 61.25 & 69.64 & 7.376 \\
\hline $\mathrm{P} 43$ & 43 & 0.1 & 8.35 & 9.38 & 0.997 & 0.997 \\
\hline Ftv170 & 171 & 0.65 & 108.28 & 96.73 & 276.1 & 276.1 \\
\hline
\end{tabular}

solve the problem of delay in achieving optimal solutions since speed is one of the hallmarks of a good algorithm [34]. In Table 6, we compare the speed of the ABO with those of the recently published Model Induced Max-Min Ant Colony Optimization (MIMM-ACO), Min-Max Ant System (MMAS) Cooperative Genetic Ant Systems (CGAS) [35], and RAI.

Table 6 displays the exceptional capacity of the novel African Buffalo Optimization (ABO) to obtain optimal or near-optimal solutions at record times. It took the $\mathrm{ABO}$ less than a second (precisely 0.95 seconds) to solve all the benchmark cases under investigation to MIMM-ACO's 232.09 seconds; MMAS's 208.88 seconds; CGAS' 452.927 seconds; and RAI's 323.4 seconds. Undoubtedly, the ABO outperformed the other algorithms in its quest to obtain results using very limited CPU time and resources. This brought about ABO's use of very few parameters coupled with straightforward fitness function. The exceptional speed of the $\mathrm{ABO}$ compared with its competitive ability to obtain very competitive results recommends the $\mathrm{ABO}$ as an algorithm of choice when speed is a factor.

\section{Comparing ABO to Neural Network Solutions to TSP}

Moreover, following the popularity and efficiency of Neural Networks in obtaining good solutions to the Travelling Salesman's Problem [36], the authors compared the performance of African Buffalo Optimization to the known solutions of some popular Neural Network algorithms. These are Angeniol's method, Somhom et al's method, Pasti and Castro's method, and Masutti and Castro's method. Comparative experimental figures are obtained from [28].

From the experimental results in Table 7 , it can be seen that only the $\mathrm{ABO}$ obtained the optimal solutions in Eil51 and Eil76, in addition to jointly obtaining the optimal result with Masutti and Castro's method in Berlin52. Aside from these, ABO outperformed the other methods in getting near-optimal solutions in Bier127, KroA100, KroB100, KroB100, KroC100, KroD100, KroE100, Ch130, Ch150, KroA150, KroB150, KroA200, KroB150, KroB200 Rat575, rl1323, fl1400, fl1400, and Rat783. It was only in Eil101 that Masutti and Castro's method outperformed ABO. This is a further evidence that $\mathrm{ABO}$ is an excellent performer even when in competition with Neural Networks methods.

\section{Conclusion}

In general, this paper introduces the novel algorithm, the African Buffalo Optimization, and shows its capacity to solve the Traveling Salesman's Problem. The performance obtained from using the $\mathrm{ABO}$ is weighed against the performance of some popular algorithms such as the Ant Colony Optimization (ACO), Particle Swarm Optimization (PSO), Artificial Bee Colony Optimization (ABO), Min-Max Ant System (MMAS), and Randomized Insertion Algorithm (RAI); some hybrid methods such as Model Induced Max-Min Ant Colony Optimization (MIMM-ACO), Hybrid Algorithm (HA), and Cooperative Genetic Ant Systems (CGAS); and some popular Neural Networks-based optimization methods. In total, 33 TSP datasets cases ranging from 48 to 14461 cities were investigated and the $\mathrm{ABO}$ results obtained were compared with results obtained from 11 other optimization algorithms and methods. The results show the amazing performance of the novel algorithm's capacity to obtain optimal or near-optimal solutions at an incredibly fast rate. The ABO's outstanding performance is a testimony to the fact that $\mathrm{ABO}$ has immense potentials in solving optimization problems using relatively fewer parameters than most other optimization algorithms in literature.

Having observed the robustness of the ABO in solving the Travelling Salesman's Problems with encouraging outcome, it is recommended that more investigations be carried out to ascertain the veracity or otherwise of this new algorithm in solving other problems such as PID tuning, knapsack problem, vehicle routing, job scheduling, and urban transportation problems.

\section{Conflict of Interests}

The authors assert that there is no conflict of interests in the publication of this research paper.

\section{Acknowledgments}

The authors acknowledge the support of the anonymous reviewers for their constructive reviews that helped to 
TABLE 7: ABO versus NN results.

\begin{tabular}{|c|c|c|c|c|c|c|c|c|c|c|c|}
\hline \multirow[t]{2}{*}{$\begin{array}{l}\text { TSP } \\
\text { instance }\end{array}$} & \multirow[t]{2}{*}{ Optima } & \multicolumn{2}{|c|}{$\mathrm{ABO}$} & \multicolumn{2}{|c|}{$\begin{array}{l}\text { Angeniol's } \\
\text { method }\end{array}$} & \multicolumn{2}{|c|}{$\begin{array}{l}\text { Somhom et al.s } \\
\text { method }\end{array}$} & \multicolumn{2}{|c|}{$\begin{array}{l}\text { Pasti and Castro's } \\
\text { method }\end{array}$} & \multicolumn{2}{|c|}{$\begin{array}{l}\text { Masutti and Castro's } \\
\text { method }\end{array}$} \\
\hline & & Best & Mean & Best & Mean & Best & Mean & Best & Mean & Best & Mean \\
\hline eil51 & 426 & 426 & 427 & 432 & 442.90 & 433 & 440.57 & 429 & 438.70 & 427 & 437.47 \\
\hline eil76 & 538 & 538 & 563.04 & 554 & 563.20 & 552 & 562.27 & 542 & 556.10 & 541 & 556.33 \\
\hline eil101 & 629 & 640 & 640 & 655 & 665.93 & 640 & 655.57 & 641 & 654.83 & 638 & 648.63 \\
\hline berlin52 & 7542 & 7542 & 7659.31 & 7778 & 8363.70 & 7715 & 8025.07 & 7716 & 8073.97 & 7542 & 7932.50 \\
\hline bier127 & 118282 & 118297 & 118863 & 120110 & 128920.33 & 119840 & 121733.33 & 118760 & 121780.33 & 118970 & 120886.33 \\
\hline ch130 & 6110 & 6111 & 6307.14 & 6265 & 6416.80 & 6203 & 6307.23 & 6142 & 6291.77 & 6145 & 6282.40 \\
\hline $\operatorname{ch} 150$ & 6528 & 6532 & 6601 & 6634 & 6842.80 & 6631 & 6751 & 6629 & 6753.20 & 6602 & 6738.37 \\
\hline rd100 & 7910 & 7935 & 7956 & 8088 & 8444.50 & 8028 & 8239.40 & 7947 & 8253.93 & 7982 & 8199.77 \\
\hline $\operatorname{lin} 105$ & 14379 & 14419 & 14452.7 & 14999 & 16111.37 & 14379 & 14475.60 & 14379 & 14702.23 & 14379 & 14400.17 \\
\hline $\operatorname{lin} 318$ & 42029 & 42101 & 42336 & 44869 & 45832.83 & 43154 & 43922.90 & 42975 & 43704.97 & 42834 & 43696.87 \\
\hline kroA100 & 21282 & 21311 & 22163.8 & 23009 & 24678.80 & 21410 & 21616.77 & 21369 & 21868.47 & 21333 & 21522.73 \\
\hline kroA150 & 26524 & 26526 & 27205 & 28948 & 29960.90 & 26930 & 27401.33 & 26932 & 27346.43 & 26678 & 27355.97 \\
\hline kroA200 & 29368 & 29370 & 30152 & 31669 & 33228.33 & 30144 & 30415.67 & 29594 & 30257.53 & 29600 & 30190.27 \\
\hline kroB100 & 22141 & 22160 & 22509 & 24026 & 25966.40 & 22548 & 22622.50 & 22596 & 22853.60 & 22343 & 22661.47 \\
\hline kroB150 & 26130 & 26169 & 26431 & 27886 & 29404.53 & 26342 & 26806.33 & 26395 & 26752.13 & 26264 & 26631.87 \\
\hline kroB200 & 29437 & 29487 & 29534 & 32351 & 33838.13 & 29703 & 30286.47 & 29831 & 30415.60 & 29637 & 30135.00 \\
\hline kroC100 & 20749 & 20755 & 20881.7 & 22344 & 23496.13 & 20921 & 21149.87 & 20915 & 21231.60 & 20915 & 20971.23 \\
\hline kroD100 & 21294 & 21347 & 21462 & 23076 & 23909.03 & 21500 & 21845.73 & 21457 & 22027.87 & 21374 & 21697.37 \\
\hline kroE100 & 22068 & 22088 & 22702 & 23642 & 24828.03 & 22379 & 22682.47 & 22427 & 22815.50 & 22395 & 22715.63 \\
\hline rat575 & 6773 & 6774 & 6810 & 8107 & 8301.83 & 7090 & 7173.63 & 7039 & 7125.07 & 7047 & 7115.67 \\
\hline rat783 & 8806 & 8811 & 8881.75 & 10532 & 10721.60 & 9316 & 9387.57 & 9185 & 9326.30 & 9246 & 9343.77 \\
\hline rl1323 & 270199 & 270480 & 278977 & 293350 & 301424.33 & 295780 & 300899.00 & 295060 & 300286.00 & 300770 & 305314.33 \\
\hline fl1400 & 20127 & 20134 & 20167 & 20649 & 21174.67 & 20558 & 20742.60 & 20745 & 21070.57 & 20851 & 21110.00 \\
\hline d1655 & 62128 & 62346 & 62599.5 & 68875 & 71168.07 & 67459 & 68046.37 & 70323 & 71431.70 & 70918 & 72113.17 \\
\hline
\end{tabular}

enhance the quality of this research paper. Similarly, the authors are grateful to the Faculty of Computer Systems and Software Engineering, Universiti Malaysia Pahang, Kuantan, Malaysia, for providing the conducive environment, facilities, and funds for this research through Grant GRS 1403118.

\section{References}

[1] J. Kennedy, "Particle swarm optimization," in Encyclopedia of Machine Learning, pp. 760-766, Springer, 2010.

[2] M. Dorigo and M. Birattari, "Ant colony optimization," in Encyclopedia of Machine Learning, pp. 36-39, Springer, 2010.

[3] E. Cantú-Paz, "A summary of research on parallel genetic algorithms," IlliGAL Report 95007, Illinois Genetic Algorithms Laboratory, 1995.

[4] D. Karaboga and B. Basturk, "A powerful and efficient algorithm for numerical function optimization: artificial bee colony (ABC) algorithm," Journal of Global Optimization, vol. 39, no. 3, pp. 459-471, 2007.

[5] J. Andre, P. Siarry, and T. Dognon, "An improvement of the standard genetic algorithm fighting premature convergence in continuous optimization," Advances in Engineering Software, vol. 32, no. 1, pp. 49-60, 2001.

[6] N. Kumbharana and G. M. Pandey, "A comparative study of ACO, GA and SA for solving travelling salesman problem,"
International Journal of Societal Applications of Computer Science, vol. 2, pp. 224-228, 2013.

[7] Z. Michalewicz and D. B. Fogel, "The traveling salesman problem," in How to Solve It: Modern Heuristics, pp. 189-224, Springer, 2000.

[8] J. B. Odili, H. Deng, C. Yang, Y. Sun, M. C. d. E. Santo Ramos, and M. C. G. Ramos, "Application of ant colony optimization to solving the traveling salesman's problem," Science Journal of Electrical \& Electronic Engineering, In press.

[9] A. Ben-Israel, "A Newton-Raphson method for the solution of systems of equations," Journal of Mathematical Analysis and Applications, vol. 15, pp. 243-252, 1966.

[10] D. P. Bertsekas, Dynamic Programming and Optimal Control, vol. 1, Athena Scientific, Belmont, Mass, USA, 1995.

[11] R. G. Ghanem and P. D. Spanos, Stochastic Finite Elements: A Spectral Approach, Courier Corporation, 2003.

[12] S. Binitha and S. S. Sathya, "A survey of bio inspired optimization algorithms," International Journal of Soft Computing and Engineering, vol. 2, pp. 137-151, 2012.

[13] F. Dressler and O. B. Akan, "A survey on bio-inspired networking," Computer Networks, vol. 54, no. 6, pp. 881-900, 2010.

[14] X.-S. Yang and S. Deb, "Two-stage eagle strategy with differential evolution," International Journal of Bio-Inspired Computation, vol. 4, no. 1, pp. 1-5, 2012. 
[15] S. K. Dhurandher, S. Misra, P. Pruthi, S. Singhal, S. Aggarwal, and I. Woungang, "Using bee algorithm for peer-to-peer file searching in mobile ad hoc networks," Journal of Network and Computer Applications, vol. 34, no. 5, pp. 1498-1508, 2011.

[16] A. R. Mehrabian and C. Lucas, "A novel numerical optimization algorithm inspired from weed colonization," Ecological Informatics, vol. 1, no. 4, pp. 355-366, 2006.

[17] M. Dorigo, G. Di Caro, and L. M. Gambardella, "Ant algorithms for discrete optimization," Artificial Life, vol. 5, no. 2, pp. 137$172,1999$.

[18] M. Clerc, Particle Swarm Optimization, vol. 93, John Wiley \& Sons, 2010.

[19] Z. Yuan, M. A. M. de Oca, M. Birattari, and T. Stützle, "Continuous optimization algorithms for tuning real and integer parameters of swarm intelligence algorithms," Swarm Intelligence, vol. 6, no. 1, pp. 49-75, 2012.

[20] D. Karaboga and B. Akay, "A survey: algorithms simulating bee swarm intelligence," Artificial Intelligence Review, vol. 31, no. 14, pp. 61-85, 2009.

[21] J. Kennedy and R. Mendes, "Population structure and particle swarm performance," in Proceedings of the Congress on Evolutionary Computation (CEC '02), vol. 2, pp. 1671-1676, IEEE, Honolulu, Hawaii, USA, May 2002.

[22] A. M. Mora, P. García-Sánchez, J. J. Merelo, and P. A. Castillo, "Migration study on a pareto-based island model for MOACOs," in Proceedings of the 15th Genetic and Evolutionary Computation Conference (GECCO '13), pp. 57-64, July 2013.

[23] M. Dorigo and L. M. Gambardella, "Ant colonies for the travelling salesman problem," BioSystems, vol. 43, no. 2, pp. 7381, 1997.

[24] M. G. C. Resende, R. Martí, M. Gallego, and A. Duarte, "GRASP and path relinking for the max-min diversity problem," Computers \& Operations Research, vol. 37, no. 3, pp. 498-508, 2010.

[25] G. Reinelt, “Tsplib95, 1995,” 2012, http://comopt.ifi.uni-heidelberg.de/software/TSPLIB95.

[26] M. Gündüz, M. S. Kiran, and E. Özceylan, "A hierarchic approach based on swarm intelligence to solve the traveling salesman problem," Turkish Journal of Electrical Engineering and Computer Sciences, vol. 23, no. 1, pp. 103-117, 2015.

[27] H. Jia, "A novel hybrid optimization algorithm and its application in solving complex problem," International Journal of Hybrid Information Technology, vol. 8, pp. 1-10, 2015.

[28] S.-M. Chen and C.-Y. Chien, "A new method for solving the traveling salesman problem based on the genetic simulated annealing ant colony system with particle swarm optimization techniques," in Proceedings of the International Conference on Machine Learning and Cybernetics (ICMLC '10), pp. 2477-2482, July 2010.

[29] S. Georg, "MP-TESTDATA-The TSPLIB symmetric traveling salesman problem instances," 2008.

[30] K. Sörensen, "Metaheuristics-the metaphor exposed," International Transactions in Operational Research, vol. 22, no. 1, pp. 3-18, 2015.

[31] J. Brest and J. Zerovnik, "A heuristic for the asymmetric traveling salesman problem," in Proceedings of the 6th Metaheuristics International Conference (MIC '05), pp. 145-150, Vienna, Austria, 2005.

[32] M. Dorigo and L. M. Gambardella, "Ant colony system: a cooperative learning approach to the traveling salesman problem," IEEE Transactions on Evolutionary Computation, vol. 1, no. 1, pp. 53-66, 1997.
[33] O. C. Martin and S. W. Otto, "Combining simulated annealing with local search heuristics," Annals of Operations Research, vol. 63, pp. 57-75, 1996.

[34] B. Baritompa and E. M. Hendrix, "On the investigation of stochastic global optimization algorithms," Journal of Global Optimization, vol. 31, no. 4, pp. 567-578, 2005.

[35] J. Bai, G.-K. Yang, Y.-W. Chen, L.-S. Hu, and C.-C. Pan, "A model induced max-min ant colony optimization for asymmetric traveling salesman problem," Applied Soft Computing, vol. 13, no. 3, pp. 1365-1375, 2013.

[36] N. Aras, B. J. Oommen, and I. K. Altinel, "The Kohonen network incorporating explicit statistics and its application to the travelling salesman problem," Neural Networks, vol. 12, no. 9, pp. 1273-1284, 1999. 

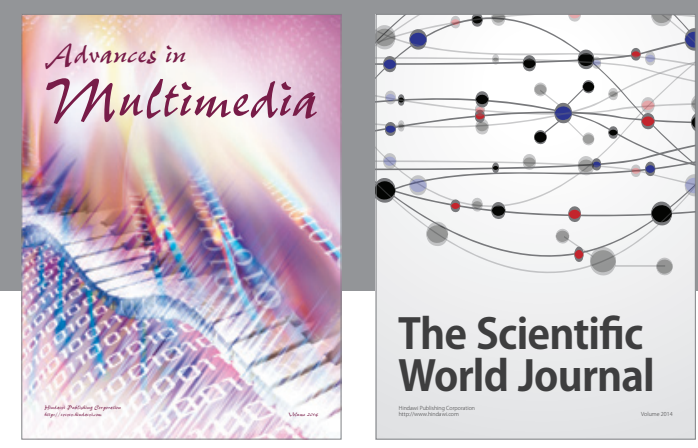

The Scientific World Journal
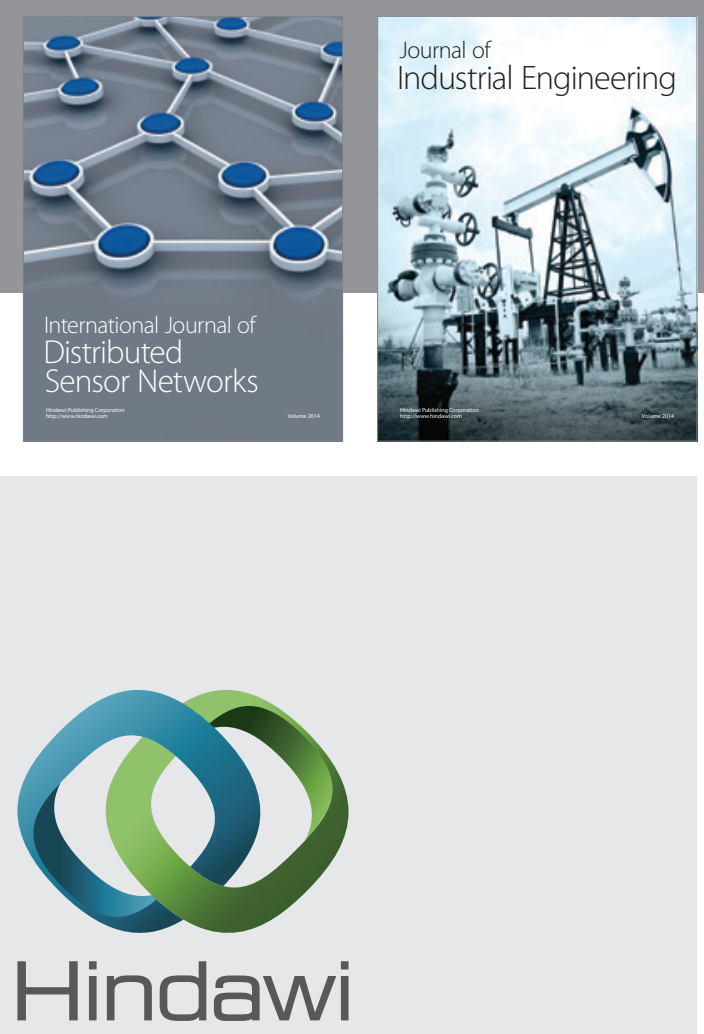

Submit your manuscripts at

http://www.hindawi.com

\section{Computer Networks} and Communications
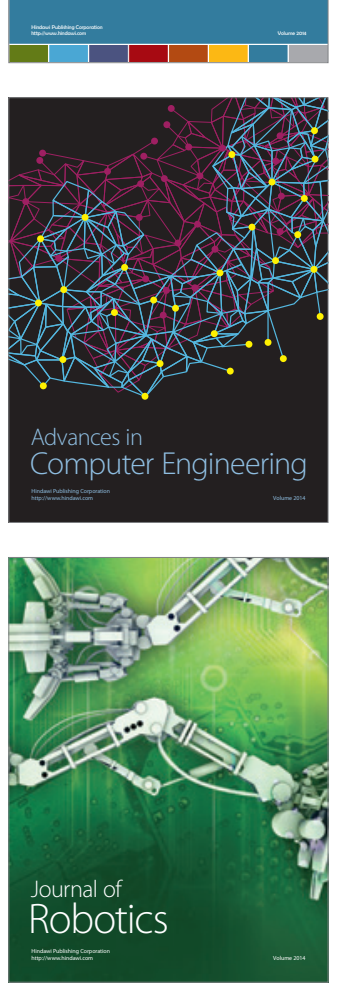
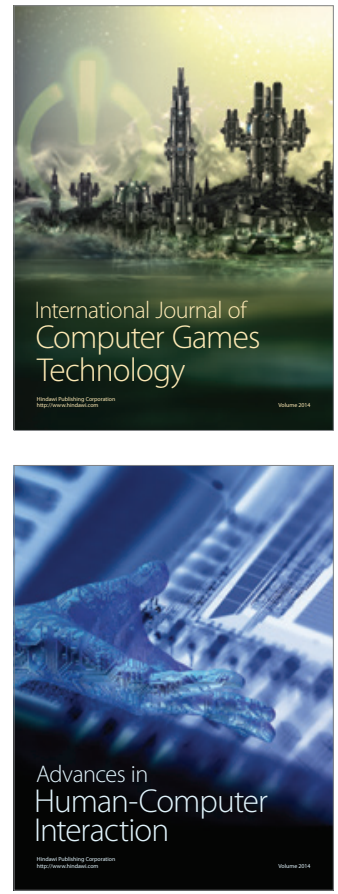
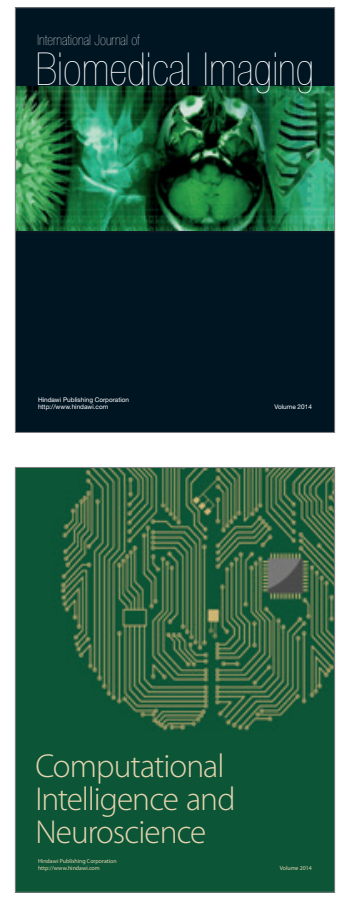
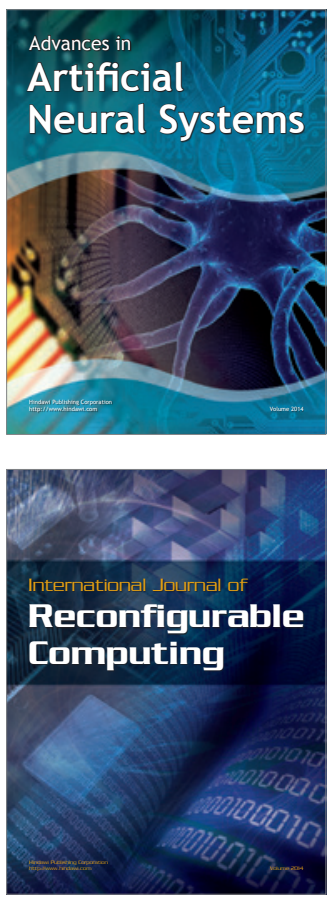
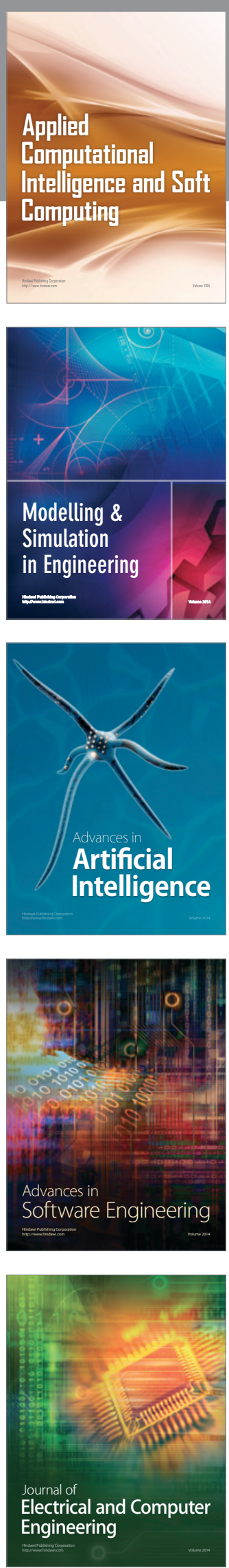